\title{
8
}

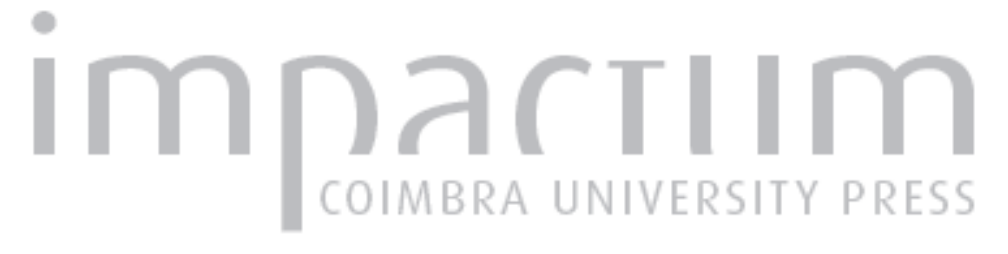

\section{Pessoa à Coimbra et Coimbra dans Pessoa}

Autor(es): $\quad$ Frias, Aníbal

Publicado por: Faculdade de Letras da Universidade de Coimbra

URL persistente:

URI:http://hdl.handle.net/10316.2/32763

DOI:

DOI:http://dx.doi.org/10.14195/0870-4112_7_16

Accessed : $\quad$ 26-Apr-2023 10:44:51

A navegação consulta e descarregamento dos títulos inseridos nas Bibliotecas Digitais UC Digitalis, UC Pombalina e UC Impactum, pressupõem a aceitação plena e sem reservas dos Termos e Condições de Uso destas Bibliotecas Digitais, disponíveis em https://digitalis.uc.pt/pt-pt/termos.

Conforme exposto nos referidos Termos e Condições de Uso, o descarregamento de títulos de acesso restrito requer uma licença válida de autorização devendo o utilizador aceder ao(s) documento(s) a partir de um endereço de IP da instituição detentora da supramencionada licença.

Ao utilizador é apenas permitido o descarregamento para uso pessoal, pelo que o emprego do(s) título(s) descarregado(s) para outro fim, designadamente comercial, carece de autorização do respetivo autor ou editor da obra.

Na medida em que todas as obras da UC Digitalis se encontram protegidas pelo Código do Direito de Autor e Direitos Conexos e demais legislação aplicável, toda a cópia, parcial ou total, deste documento, nos casos em que é legalmente admitida, deverá conter ou fazer-se acompanhar por este aviso.

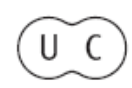




\section{Sociedade em Tumulto}

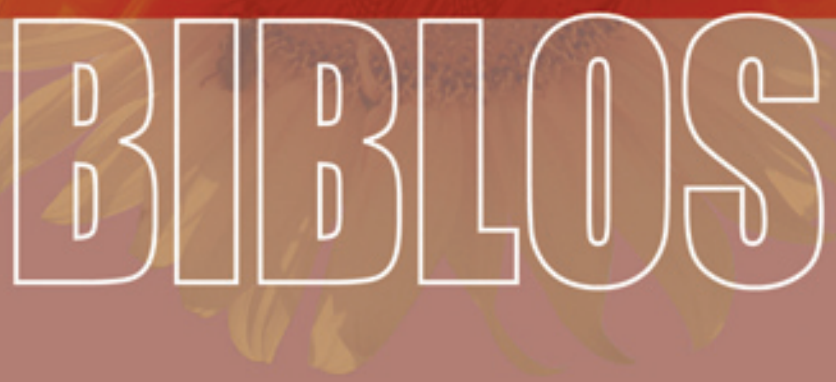

REVISTA DA FACULDADE DE LETRAS

UNIVERSIDADE DE GOIMBRA 


\author{
Aníbal Frias ${ }^{1}$ \\ Universidade de Coimbra
}

\title{
PESSOA À COIMBRA ET COIMBRA DANS PESSOA
}

\section{RESUMO}

A relação entre Pessoa e Coimbra não se limita à revista Presença. O poeta foi convidado em 1919 a ensinar na Universidade de Coimbra. Os seus escritos circulam na "cidade dos doutores" sob a forma de iconografias, especialmente símbolos e heróis da Mensagem, e instrumentalizados pelo regime salazarista. A relação entre o escritor e Coimbra também pode ser invertida na medida em que Pessoa encena, muito cedo na sua obra, personagens e rituais praxísticos influenciados pela cultura estudantil, e nunca identificados.

Palavras-chave: Pessoa, Coimbra, estudantes, praxe, estética nacionalista.

\begin{abstract}
The relationship between Pessoa and Coimbra is not limited to the periodical Presença. In 1919, the poet was invited to teach at the University of Coimbra. His writings circulated in this city in the form of pictures, especially some related to the symbols and heroes of Mensagem, and were instrumentalized by the Salazar regime. The relationship between the writer and Coimbra can also be reversed, insofar as Pessoa features, very early in his work, characters and rituals influenced by the student culture, which have never been identified.
\end{abstract}

Keywords: Pessoa, Coimbra, students, hazing, nationalist aesthetics.

Si Fernando Pessoa n'est jamais allé à Coimbra, ses vers et sa prose y sont imprimés, en particulier dans la revue Presença, son nom circule parmi les universitaires et sa notoriété grandissante avec lui. Jusqu'à une imagerie nationaliste détournée de sa poésie. A l'inverse, la Coimbra étudiante est présente en filigrane dans des écrits du poète.

\footnotetext{
${ }^{1}$ Boursier post-doctoral de la Fundação para a Ciência e a Tecnologia; chercheur au CEIS20 - Université de Coimbra. 
Dans un premier moment, nous éclairerons le contexte historique de l'invitation adressée à Pessoa pour qu'il vienne enseigner à l'Université de Coimbra. Ensuite, à partir de son œuvre, nous analyserons les références aux cultures étudiantes, spécialement celles situées sur la Alta, la colline studieuse baignée par le fleuve Mondego. Cette question, jamais traitée, nous conduira à réévaluer la genèse de la problématique de l'hétéronymie. Enfin, nous identifierons les formes et les raisons d'une projection iconographique inspirée de Mensagem dans la Faculté de lettres de la Cité Universitaire de Coimbra, sous l'Estado Novo. La relation de Pessoa et de Coimbra peut donc être réversible et entendue de trois manières: en termes de contacts personnels, de références littéraires ou bien de présences imaginaires.

\section{L'invitation}

Pessoa à Coimbra? L'opportunité s'est du moins présentée. Selon le témoignage de son cousin Eduardo Freitas da Costa, Fernando Pessoa a été convié par le reitor de l'Université de Coimbra. Mais il "a refusé obstinément les invitations réitérées que lui a adressé le dr. Coelho de Carvalho, alors président de notre première Université, afin d'occuper la chaire de langue et littérature anglaises de la Faculté des lettres de Coimbra" ${ }^{2}$. La mention de cet épisode (référé sans autres détails) est destinée à contrecarrer l'excessive vision misérabiliste et le manque de sens pratique qualifiant un poète supposé inapte pour la vie. Ce portrait romantique, devenu "légendaire", est forgé par la biographie de João Gaspar Simões, parue un an plus tôt ${ }^{3}$. Malgré une telle proposition inespérée, Pessoa choisit de se maintenir dans sa condition d'"employé de commerce" préposé à la "correspondance commerciale en langues étrangères", qui lui permet, tant bien que mal, de se consacrer à l'essentiel: son œuvre.

Cette information, si elle est peu rappelée par les spécialistes, reste par elle-même assez obscure. Qu'en est-il des raisons de cette invitation? Il est possible d'en cerner le contexte historique et les circonstances sociales. José Joaquim Coelho de Carvalho est nommé, par le gouvernement républicain, reitor intérimaire de l'Université de Coimbra en mars 1919.

\footnotetext{
${ }^{2}$ E. Freitas da Costa, Fernando Pessoa. Notas a uma biografia romanceada (Lisbonne, Guimarães, 1951) 63.

${ }^{3}$ J. Gaspar Simões, Vida e obra de Fernando Pessoa (Lisbonne, Livraria Bertrand, 1950, 4e éd. 1979). 
Titulaire d'un simple bacharelato en droit, le nouveau reitor n'appartient pas au corps professoral puisqu'il a fait sa carrière dans la diplomatie depuis la fin du XIXe siècle. Il a été choisi pour diriger l'Université par le ministre de l'Instruction de l'époque, Leonardo Coimbra, philosophe républicain et cofondateur de la Renascença Portuguesa. Le nouveau reitor est lui-même un républicain "préhistorique", comme il se désigne dans son allocution de prise de fonction ${ }^{4}$. Il arrive dans une Université agitée par la tourmente. Ce climat est provoqué par la suspension, en mars, de professeurs antidémocrates de la Faculté de droit, dont le jeune Salazar, accusés de transformer leur chaire en tribune politique et religieuse et d'avoir soutenu la récente révolte monarchiste de Porto, survenue en début d'année (19/01/1919). Il ne reste que trois mois en poste, étant rapidement contesté pour avoir manqué de loyauté envers la corporation universitaire, en faisant le jeu du gouvernement qui a tenté de transférer la Faculté de lettres vers Porto.

Nous pouvons retenir plusieurs ingrédients qui éclairent l'invitation faite à Pessoa. En premier lieu, le moment où intervient cette demande: elle se situe dans une très courte période de trois mois, quatre années après le moment turbulent d'Orpheu, et quelques mois seulement après le choc provoqué par l'assassinat de Sidónio Pais. Autrement dit, à une étape de la vie de Pessoa où il a déjà publié des textes littéraires et d'opinion importants, et commence à être connu des milieux intellectuels et culturels. Il se trouve que, dans son discours inaugural, Coelho de Carvalho fait référence à la "convivialité" qu'il a l'habitude d'entretenir avec les "intellectuels et artistes". Cela s'explique par le fait qu'il est lui-même un homme de lettres, étant traducteur et dramaturge. Sa connaissance de Pessoa remonte au temps d'Orpheu. En témoigne une annotation du poète dans son journal en date du 7 décembre 1915: "Vitoriano Braga m'a parlé ce matin du désir de Coelho de Carvalho de me voir traduire le Faust; mais, hélas!, le gain est hypothétique et n'est seulement perçu qu'après le travail accompli!"'5.

La fréquentation des créateurs à cette époque est forcément le fait de quelqu'un d'étranger à l'Université, celle-ci étant par trop repliée

4 'Sessão de 20 de Março de 1919. Posse que o Ex.mo Dr. Joaquim Coelho de Carvalho tomou do lugar de Reitor interino da Universidade de Coimbra', in M. A. Rodrigues (éd.), A Universidade de Coimbra no Século XX (Coimbra, Publicações do Arquivo da Universidade de Coimbra, 1988) 34-39.

${ }^{5} \mathrm{~F}$. Pessoa, Escritos autobiográficos, automáticos e de reflexão pessoal (Lisbonne, Assírio e Alvim, 2003 - édition de R. Zenith) 173. 
sur son organisation corporative et son inertie. Si l'on rapproche cette sociabilité culturelle affichée par le reitor de son itinéraire professionnel antérieur, celui de consul, qui l'a conduit en Afrique (du Sud?), on peut avec raison supputer que, dans le petit univers lettré de Lisbonne et, plus encore, dans le microcosme sélectif et mondain de la diplomatie portugaise où circule le beau-père de Pessoa, l'invitation dirigée à l'écrivain par Coelho de Carvalho devait probablement relever d'une cooptation favorisée et du geste "personnel". Pessoa lui-même, dès son retour de Durban en 1905, s'était inscrit dans le "cours pour les carrières consulaire et diplomatique". A l'appui de cette interprétation, nous pouvons avancer un autre indice présent dans les actes universitaires: il s'agit de la décision arbitraire reprochée au reitor, car elle va à l'encontre du règlement, d'avoir effectué une "nomination extraordinaire de professeurs, sans audience préalable de la Faculté"6.

\section{Des références en miroir}

Le deuxième type de relation qui lie Pessoa à Coimbra est d'ordre littéraire. Nous retiendrons deux écrits de jeunesse: $O$ Palrador et $O s$ rapazes de Barrowby. Notre angle d'analyse fera apparaître des éléments caractéristiques du monde lettré de Coimbra. Nous proposerons, pour le second texte, une nouvelle interprétation fondée sur les cultures étudiantes.

\section{O Palrador: Dr. Caloiro et Pad Zé}

Il existe dans les écrits de Fernando Pessoa une référence implicite à Coimbra, quoique assurée, comme nous le montrerons. Elle est visible dans un article intitulé "La pêche des perles", signé par un certain Dr. Caloiro. Il est publié dans un petit journal domestique manuscrit et écrit en portugais: $O$ Palrador ${ }^{7}$; il comporte différents numéros de 1902, 1903 et 1905. L'article en question est paru dans le numéro 5 du 22 mars 1902. Cette feuille est entièrement composée par un "Pessôa" de 13 ans. Ses multiples signatures varient selon les statuts incarnés des "journalistes": rédacteurs, chroniqueurs, directeur littéraire, administrateur, etc. Teresa

6 'Cópia da Representação votada na reunião dos professores de 25 de Maio de 1919 "Universidade de Coimbra”, in M. A. Rodrigues (éd.), A Universidade de Coimbra no Século XX (Coimbra, Publicações do Arquivo da Universidade de Coimbra, 1988) 51.

${ }^{7}$ Publié par T. Rita Lopes, Pessoa por conhecer, vol. II (Lisbonne, Editorial Estampa, 1990) texte 101. 
Rita Lopes range le nom de Dr. Caloiro parmi les proto-hétéronymes et les personnages littéraires qui totalisent plus de soixante-dix dramatis personae inventés.

Que signifie le syntagme "Doutor Caloiro", employé ici comme nom propre? Au Portugal, on le sait, le caloiro (bizut) désigne dans l'argot étudiant, depuis le début du XXe siècle jusqu'à aujourd'hui, l'élève inscrit en première année universitaire, et appelé auparavant depuis le Palito Métrico (1792) novato. Associé, comme dans notre cas, à "doutor", il forme une polarité structurellement antithétique: le "doutor" c'est l'"ancien" dans les études, par opposition au "nouveau". Le mot "doutor" est un détournement parodique, par l'Academia, du titre et de l'appellation officiels du professeur docteur de l'Université. Cette appropriation créative lui confère, en même temps qu'une valeur ludique, une fonction rituelle. La plupart des constituants culturels de l'Academia de Coimbra sont organisés autour d'un principe hiérarchique, à l'image de l'Université, qui commande la soumission des caloiros aux "doutores" dans une configuration de relations ludico-rituelles.

Ce qui est tout à fait habile chez le très jeune Fernando, c'est que, non seulement il connaît le sens ordinaire de ces termes, mais surtout il a compris qu'ils ne fonctionnent que dans une sorte de complementio oppositorum. Ce n'est pas tout. Les chercheurs ont remarqué l'apparition précoce du phénomène hétéronymique chez Pessoa lié à son art de fingir. Or, ce qui n'a pas encore été signalé, c'est que surgit très tôt, et en parallèle, le jeu des oxymores si caractéristique de son style. On en a justement un exemple parfait avec l'unité sémantique aux pôles contrastés, "Dr. Caloiro".

Dans le Palrador, plusieurs personnages qui intègrent la première "coterie" pessoenne possèdent un surnom en plus de leur "vrai" nom. Le surnom se trouve accolé à leur identité (imaginaire) puisqu'il fait office de signature hétérographe, qui authentifie et identifie. C'est le cas de Pad Zé qui est le surnom de Pedro da Silva Salles ( $c f$. infra). Il est d'ailleurs un jumeau en pseudonymie de Roberto Kóla puisque ce dernier s'est également approprié une telle appellation. Pad Zé a en charge des charades, des anecdotes et autres "énigmes pittoresques". Ce dédoublement dans le dédoublement correspond à l'opération par laquelle Pessoa "s'autre" - très tôt on le voit - en un auteur proto-hétéronyme, lequel à son tour adopte un pseudonyme. L'autonyme et le personnage

${ }^{8}$ T. Rita Lopes, Pessoa por conhecer, vol. I (Lisbonne, editorial Estampa, 1990) 167. 
littéraire peuvent éventuellement coexister en entrelaçant la réalité et la fiction, où le masque intervient comme l'ombre portée du moi pessoen: ainsi, Pessoa et le Dr. Pancrácio se partagent-ils la direction du petit journal manuscrit A Palavra (1902) qui suit les premiers numéros du Palrador. En cette période qui précède de douze ans le "jour triomphal", cette altérité nominale est de consistance littéraire.

Après le 8 mars 1914, le dédoublement fictionnel va plus loin dans la complexité et dans l'illusion de réalité. A partir de ce moment, les personnages fictionnels hypostasiés acquièrent une consistance ontologique, avec les hétéronymes ou "poétodrames", et un corps textuel, avec les "poémodrames". En outre, ce qui s'annonce déjà en 1902, et qui sera par la suite davantage systématisé avec le "dialogue en famille" et les citations "intra-textuelles" entre hétéronymes (orthonyme compris), c'est une autonomisation des acteurs de la "coterie". Cette quasi-indépendance est obtenue par un effet de réel travaillé au sein de la fiction. On le voit à la biographie que deux des personnages font d'un tiers collaborateur brésilien, le poète Eduardo Lança, tout aussi inexistant que les deux autres. On le voit encore aux dédicaces que se font entre eux certains des "journalistes" du Palrador, notamment entre Diabo Azul et Pad Zé qui se rendent par là un mutuel hommage. Tout se passe comme si ces êtres de papier établissaient une relation de complicité directement entre eux. En existant dans "l'espace incolore mais réel du rêve", ils agissent comme à l'insu de leur créateur Pessoa, vivant en lui et "indépendamment de [lui]"10.

Il nous reste à établir le lien direct sinon de Pessoa, du moins de son Dr. Caloiro, avec Coimbra, car il n'est pas formulé. Plusieurs raisons encouragent à faire un tel rapprochement. La date de la publication constitue un premier fait révélateur: en 1902, le Portugal ne compte qu'une seule Université, celle de Coimbra, alors qu'une telle institution n'émerge à Lisbonne et à Porto qu'en 1911, où auparavant il a existé des écoles supérieures ou bien, dans la capitale, un Cours supérieur de lettres dans lequel, du reste, Pessoa s'inscrit en 1905-1907. Cet argument n'est toutefois pas une preuve suffisante, étant donné que des aspects de la coutume étudiante se retrouvent dans les deux autres principales villes du pays, y compris dans les lycées nationaux. Un second indice, présent dans tous les numéros du Palrador de 1902, nous pousse à retenir Coimbra comme la seule provenance possible d'une double référence aux traditions étudiantes

${ }^{9}$ J.A. Seabra, Fernando Pessoa ou o Poetodrama (Lisbonne, IN-CM, 1988).

${ }^{10}$ Ces deux expressions sont tirées de la lettre sur la genèse des hétéronymes du 13 janvier 1935. 
(Praxe académica), introduite par Pessoa dans son écrit de jeunesse. Il s'agit d'un autre "auteur" proto-hétéronymique, collègue du Dr. Caloiro: Pedro da Silva Salles, dit Pad Zé, qui assume la fonction de rédacteur. Ce que l'on retiendra de ce personnage, c'est d'abord son surnom: Pad Zé.

Durant la même période, un Pad'Zé, alias Alberto Costa, fréquente l'Université de Coimbra entre 1895 et 1904. A son arrivée sur la "colline sacrée", il acquiert une telle popularité qu'elle finit par dépasser l'aire urbaine de l'Academia. Cet étudiant est amateur de blagues, de canulars et de tertúlias; avec son condisciple Afonso Lopes Vieira, il sort au tournant du siècle une revue fugace dédiée à l'humour, aux vers et à la prose ${ }^{11}$ (à l'égale du Palrador). Ses faits et gestes, aussi subtils que futiles, ont été consignés dans ses Mémoires anecdotiques à succès, édités en 1905. Alberto Costa compte parmi les "doutores" qui ont improvisé, en forme de parodie, le défilé festif étudiant du Entero da sebenta, en 1899, d'où dérive l'actuelle Queima das fitas, grand cortège annuel étudiant composé de dizaines de chars aux contours carnavalesques. Les épisodes les plus drôles et inventifs de ce cábula (étudiant bohème, par opposition à l'urso - l'élève modèle) sont parfois relayés par la presse locale et nationale. Ils ont survécu dans la tradition écrite de l'Academia et dans les mémoires des futricas (la population non universitaire de la Baixa). Au point que, en 1936, les nouvelles générations étudiantes, entre saudosismo et traditionalisme, appuient la mairie qui souhaite attribuer une rue à Pad'Zé, figure idéalisée de l' "étudiant de Coimbra".

L'esprit curieux et aiguisé du jeune Fernando permet de conclure qu'il a pu avoir connaissance des prouesses de ce personnage dont il est le contemporain. D'autant que notre poète en herbe se trouve à Lisbonne à l'époque de la fabrication du Palrador, à quelques semaines de son départ avec sa famille vers les Açores. L'humour zébré d'ironie du Pad'Zé de Coimbra et son surnom, devenu nom identitaire, ne pouvaient qu'éveiller la créativité du Pad Zé pessoen... à une apostrophe près $^{12}$. Cette interférence du réel et de la fiction se situe à la limite de

${ }^{11}$ J. Mendes Rosa, Pad'Zé: o cavaleiro da utopia, edição da Junta de Freguesia de Aldeia de Joanes, 2000.

${ }^{12}$ Dans la notice consacrée au personnage "Diabo Azul" (in F. Cabral Martins (coord.), Dicionário de Fernando Pessoa e do Modernismo português, Lisbonne, Caminho, 2008, p. 219), M. Parreira da Silva signale l'existence de ce nom, depuis au moins l'année 1900, dans le journal O Pimpão; elle indique également la présence, mais sans préciser de date, de ceux de Pad Zé et de Dr. Pancrácio. Tous ces êtres sont des "collaborateurs" de $O$ Palrador, et sous ces mêmes appellations. M. Parreira da Silva 
l'intertextualité, avec la similitude existant entre les vannes et pitreries de l'un et la spécialité rédactionnelle de l'autre: charades et autres "énigmes pittoresques"13.

\section{Os rapazes de Barrowby}

Ce court texte en prose apparaît en juillet 1903 dans un autre numéro d' $O$ Palrador ( $\mathrm{n}^{\circ} 1$, nouvelle série). Il est attribué au personnage littéraire Adolph Moscow, un anglais lusographe. Il s'agit du premier roman publié sur les "quatre romans intéressants" annoncés. Cette narration en portugais se divise en deux parties conjointes: la description et localisation (à l'aide d'un plan) du bourg inventé de Barrowby et de son collège de garçons (chap. I), sont suivies d'un développement consacré à l'arrivée de trois nouveaux élèves, dont la physionomie se trouve détaillée, et de leur réception chahutée par les plus anciens (chap. II). Cette histoire met en scène des aspects de la coutume étudiante qui sont fort proches de ceux en vigueur à Coimbra jusqu'à l'actualité. Aussi, cet univers nous aidera-t-il à éclairer le texte pessoen.

Les analyses approfondies de Os rapazes de Barrowby demeurent inexistantes, en dehors de quatre références significatives. La première date de la fin des années 60, quand Hubert Dudley Jennings signale pour

soutient que c'est Henrique Rosa qui, dans $O$ Pimpão, se cache derrière ces pseudonymes. Pessoa était très lié à ce poète, frère de son beau-père. Manuela Nogueira, la nièce de Pessoa, avance l'idée, sur la base d'une consultation des numéros de $O$ Pimpão, que la plupart des proto-hétéronymes de $O$ Palrador sont déjà présents dans ce journal national, avec des charades, des mots croisés et des mini-contes. C'est le cas de Pad Zé, de Dr. Caloiro et de Diabo Azul. Ce dernier personnage écrit notamment des contes, en grande quantité et de façon intensive en 1901-1902 (lors du séjour au Portugal du poète); ce qui suggère, au regard de la variété de leur style et du contenu des histoires, qu' "il est hautement improbable qu'ils aient été écrits par la même personne”. (M. Nogueira, 'A influência de Henrique Rosa em Fernando Pessoa e o mistério do Diabo Azul': in J. Pizarro (org.), Fernando Pessoa: o guardador de papéis, Lisbonne, 2009, 32).

${ }^{13}$ Un autre témoignage indique l'intérêt précoce de Pessoa pour les cultures étudiantes: il s'agit de sa traduction inachevée (référée dans son journal de 1906) du long poème romantique, mettant en scène les amours tragiques d'un étudiant, composé par l'espagnol José de Espronceda (1808-1842), El estudiante de Salamanca. Cette tradition castillane met en scène un étudiant pícaro de Salamanque, sorte de moderne goliard bohème, pauvre, débrouillard et embobineur, vivant de vin, d'amour et de vers fredonnés. Autant d'usages et d'images qui se retrouvent à Coimbra. 
la première fois l'existence de cet écrit, sur lequel il reviendra plus tard, quoique superficiellement, dans sa thèse ${ }^{14}$. Dans sa dissertation, il met en rapport cette histoire, dont les péripéties renvoient au monde de l'école, et l'expérience personnelle de Pessoa dans la High School de Durban (personnages, brimades). La deuxième analyse est celle d'António Sabler ${ }^{15}$; en fait elle se résume à une brève présentation du récit inédit qui vient à la suite. Porté par le sous-titre, "Chronique humoristique", il centre son propos sur les quelques passages de l'histoire qui relèvent de l'humour anglais, en signalant après Jennings l'influence de Dickens. Yara Frateschi Vieira signe la troisième étude ${ }^{16}$. Elle révèle la présence dans la culture anglo-saxonne, qui est pétrie de morale victorienne, d'un imaginaire prégnant et de discours, notamment littéraires, qui associent souffrance et érotisme. Pour l'auteur, cet imaginaire et la pratique de la flagellation "pédagogique" en vigueur dans les écoles anglo-saxonnes les plus sélectives, ont influencé l'univers fantasmatique et sadomasochiste de Pessoa. Une telle correspondance se retrouve, selon l'indication (sans démonstration) de Yara F. Vieira, dans Os rapazes de Barrowby, avec les brimades et insultes adressées aux nouveaux collégiens qui sont soumis, selon l'usage du fagging, à l'autorité et à la protection des plus "grands". Le jeune Fernando au physique frêle a pu assister en voyeur à ces fréquentes scènes publiques, voire en tant que sujet soumis à la discipline sévère et à l' "obéissance à des Maîtres qui ne tolèrent ni ne pardonnent", et prompts à infliger des punitions corporelles aux réfractaires. L'auteur laisse de côté l'élément central de la tradition étudiante. Or il n'y a pas que le sadomasochisme de l'éducation anglaise qui a dû marquer le jeune Fernando, mais sans doute aussi, dans son prolongement, les rites de bizutage (praxes) dans leur forme violente (pledge, hazing). La praxe académica découle de la hiérarchie instituée entre les aînés et les cadets qui, ensemble, sont ordonnancés selon le temps de présence dans l'établissement; en conséquence de quoi les novices sont soumis aux "anciens". Ce schéma se retrouve ailleurs, avec des variantes. Une partie des rituels des colleges est réglée par un code des relations où les protections concédées aux plus petits se paient en services matériels et sexuels (hazing). Aussi, les scènes qui ressemblent à

${ }^{14}$ H.D. Jennings, Os dois exílios. Fernando Pessoa na Africa do Sul (Porto, Centro de Estudos Pessoanos, s.d.) 80-84.

${ }^{15}$ A. Sabler, 'The man who liked Dickens', Persona 9 (1983) 47-49.

${ }^{16}$ Y. Frateschi Vieira, Sob o ramo da bétula. Fernando Pessoa e o erotismo vitoriano (São Paulo, editora da Unicamp, 1989) 23-24. 
des actes sadomasochistes, pour un observateur extérieur, s'ancrent dans ces pratiques ludo-rituelles, étant en réalité une dimension du processus de praxe. Enfin, la dernière référence est due à Jerónimo Pizarro ${ }^{17}$. Il souligne l'importance précoce chez Pessoa de son intérêt littéraire et personnel pour la physiognomonie, en particulier ici la forme du nez (caricaturé à travers paroles et dessins), qui établit un lien nosographique entre l'apparence physique du sujet, et d'abord la morphologie de son visage, et un trait pathologique de nature psychomédicale.

Toutes ces interprétations de Os rapazes de Barrowby ne retiennent, chacune à sa façon, qu'une dimension particulière et extrapolée du texte, à savoir, tour à tour: l'expérience scolaire de Pessoa, l'humour anglais, l'érotisme sadomasochiste, la physionomie du nez. La réduction du contenu du texte se double d'un autre réductionnisme de type méthodologique. Ces approches ne proposent pas une explication globale, à partir de la cohérence du texte et de la totalité des faits et gestes qui s'y déploient; certaines ont en outre tendance à rabattre sans précaution le littéraire sur le littéral supposé biographique. L'explication la plus plausible d'une œuvre n'est-elle pas celle qui, en partant des éléments textuels sans perdre de vue le texte comme un tout, avance une hypothèse explicative dont la pertinence se vérifie au fait qu'elle prend en compte un maximum de composants de cette œuvre, tout en dégageant l'unité structurante qui relie ces composants les uns aux autres afin d'établir, in fine, la signification d'ensemble?

Dans cette perspective, l'hypothèse que nous soumettons à vérification dans l'espace fictionnel de Os rapazes de Barrowby, est celle qui voit d'abord et surtout dans cette histoire un rituel de praxe entre collégiens. Autrement dit, la référence englobante que l'on retient, c'est celle du milieu culturel et des sociabilités scolaires, marqués par des habitudes et des habitus sui generis et un entre-soi adolescent. Une phase de la vie qui coïncide justement - sans s'y réduire - avec l'âge et peut-être les soucis afférents au jeune Pessoa.

Les interactions entre les élèves se consolident d'autant mieux qu'elles se déroulent en un lieu clos et isolé du cadre urbain. Le mode d'organisation pyramidal et autonome, l'architecture panoptique et

17 J. Pizarro, Fernando Pessoa: entre génio e loucura (Lisbonne, IN-CM, collection "Estudos", vol. III, 2007) 19-23. La courte notice sur A. Moscow de M. Parreira da Silva s'en tient à ce point de vue physionomiste: in F. Cabral Martins (coord.), Dicionário de Fernando Pessoa e do Modernismo português (Lisbonne, Caminho, 2008) 495. 
la discipline encadrée, font penser à une "institution totale", au sens d'Erving Goffman. L'établissement éducatif est définit comme étant masculin et réservé, selon la précision appuyée de Pessoa, aux seuls internes. Toutes ces caractéristiques rappellent le college anglais du type Public School, dont le nom Barrowby semble être un composé de deux colleges réels fameux, et sûrement connus de Pessoa: ceux de Harrow et de Rugby. En échappant toutefois aux coordonnées géographiques, village et collège de Barrowby existent en quelque sorte dans un espace "autre", hétérotopique.

Comment se manifestent alors les usages scolaires dans $O s$ rapazes de Barrowby? Le titre principal de ce roman est déjà par lui-même suggestif. Ce récit se réfère à un lieu à la réalité probable, encore que non prouvée puisqu'il est sans repères - étant, pour cela même, imaginé. La traduction en anglais, permise par l'unité formée par la langue et le (non-)lieu, donne "The boys of Barrowby". Si l'on retient que Pessoa a toujours valorisé le sens "chiffré" qu'il dépose dans les mots, jusqu'à l'intérêt pour les charades qui abondent dans $O$ Palrador, d'où est issue cette création; et si, d'autre part, l'on sait le soin avec lequel il choisit les noms des (proto-)hétéronymes, dont la plurivocité codée recouvre une identité profonde, - il est permis de voir dans "The boys of Barrowby" plus qu'une localisation de personnes en un endroit donné. Outre un écho aux deux colleges mentionnés, ne peut-on retenir une autre référence cryptée aux new boys ou newbies (en retenant des lettres de Barrowby), désignant respectivement les initiés et les caloiros? Cette remarque est renforcée par le sous-titre du second chapitre - celui qui concentre le scénario principal de l'histoire: Os recem-chegados, les "nouveaux-venus" au collège. Une telle précision indique de surcroît que ces jeunes recrues vont être au centre des attentions de la part des "anciens" comme au cœur du scénario.

Une rapide lecture des scènes de moquerie et de raillerie décrites à la suite, dès l'arrivée des néophytes, montre à l'évidence qu'elles ne s'arrêtent pas au nez. D'autres organes ou parties du corps sont visés: la taille, les yeux, les cheveux, la corpulence, la face et la bouche font également l'objet de descriptions et d'invectives. Les insultes sont ritualisées dans la mesure où, normalement, elles sont inoffensives dans un contexte de praxe: ce sont des joutes verbales ou, comme dit le texte, des "batailles d'esprit". Elles sont habituellement contrôlées par le groupe et formalisées par les règles qui circonscrivent ces pratiques ludiques; leur sens littéral se trouve comme neutralisé et mis 
à distance ${ }^{18}$, notamment par le rire qui est signalé plus d'une fois: "Tu as compris?, demandait [à un caloiro] Mel en riant aux éclats", "Mel et Don sourirent", "Don...riait sans s'arrêter". La panoplie des brimades verbales englobe des situations de contrainte psychologique. C'est le cas lorsque, face à la provocation orale de Gyp (l'un des "anciens"), sa victime lui répond, tremblant jusqu'à la voix: "Je ne veux faire aucun mal, répondit de peur Zacharias... Je voulais seulement demander où se-se-se trouve le d-d-dortoire". Le pouvoir intra-scolaire des plus grands se vérifie à travers des marques d'autorité, tel Gyp "à la langue fort sévère" et "les sourcils chargés" d'une agressivité toute théâtrale. Certains élèves soumettent leurs subordonnés plus jeunes, appelés fags, à un jeu ritualisé de services et de sévices en tous genres (fagging). D'autres situations d'obéissance à l'ordre régnant des "anciens" sont visibles. Ainsi, tel aîné mime l'attitude austère des policiers ou des maîtres: Gyp, devant le caloiro Zacharias, "retire de son cartable un cahier et un crayon et se donna un air important; "moi je suis une personne importante ici dans le collège et je veux savoir parbleu ce que tu veux aller faire dans les dortoirs à l'étage'. Gyp adopta une attitude sévère et sembla disposé à écrire". Les gestes de mansuétude de la part du puissant sont encore la manifestation magnanime de son charisme et de sa bonne volonté envers les plus petits: "Gyp lui donna [à Zacharias] un coup de poing sur le nez et lui dit avec un air imposant que pour cette fois il le laissait partir sans le punir, car il était jeune".

Les vétérans comme les newbies assument leur rôle respectif dans ce psychodrame joué à l'échelle scolaire, à la façon d'un "faire semblant" sérieux et surréel qui dramatise les gestes, les paroles et les effets. Et si la confrontation rituelle entre le vétéran Gyp et le caloiro Ralph Tig tourne mal, en débouchant sur une scène inhabituelle de pugilat, elle ne cède pas pour autant à la violence débridée. Le recours extrême à la force exprime encore la structure hiérarchique qui différencie les anciens des nouveaux, étant - si cela s'avère nécessaire - rétablie à coup de poings. D'une certaine façon, le fait d'en venir aux mains prolonge la fonction "pédagogique" assumée par les plus grands. C'est en effet en terme d'"apprentissage" que Gyp se lance

${ }^{18}$ Pour les questions relatives à la signification pragmatique des actes de praxe et à leur cadre d'observation à Coimbra, je renvoie à mon doctorat d'ethnologie: A. Frias, Le monde universitaire et la Praxe académica au Portugal. Traditions étudiantes et cultures académiques à l'Université de Coimbra (Université Paris X Nanterre, vol. 2, 2003) 576-586. 
dans la bagarre avec le caloiro qui l'a défié dans son autorité: "Je vais lui apprendre à ne pas être effronté", dit Gyp agacé. Au cours de cette bagarre, comme aussi lors des brimades rituelles, les représentants de l'école sont absents. Cet effacement volontaire souligne une large acceptation de ces pratiques, longtemps associées à la virilité et même à l'éducation physique et psychique des garçons; il arrivait que les aînés s'occupent des plus jeunes en tant que prefects, exerçant sur eux par délégation une autorité morale, sinon physique, avec l'accord des professeurs. Ce retrait des adultes souligne, par contraste, la forte autonomie des usages scolaires et l'autorégulation des élèves. Pourquoi en effet intervenir puisque les aînés reproduisent sur les cadets, dotés d'une légitime procuration, ce que les maitres infligent à leurs élèves: remontrances, punitions, soumissions, flagellations "pédagogiques"? C'est pourquoi nous pouvons voir dans cette praxe anglaise historique, bien que romancée, un élément "positif" de l'éducation, aussi ordinaire et fonctionnel que les leçons, les livres ou l'activité sportive.

La conséquence de la brigue citée plus haut entre un "ancien" et un novice s'explique pour deux raisons. D'abord parce que le nouveau, au lieu de se taire en signe de respect, comme l'exige le code hiérarchique, a osé provoquer un plus grand que lui. En effet, après s'être vu interpellé d'un "tête de bouteille brisée, va!", il lance au vétéran sur le même ton: "Espèce d'Yeux de fenêtre!". Suprême défi, la victime retourne avec talent contre son auteur l'insulte reçue, gagnant ainsi du tac au tac à cette "épreuve orale", qui demande une certaine réactivité, une agilité d'esprit et une dose de talent personnel: "Et, dis-moi, si tu ne veux pas d'un morceau de tripe pourri dans le collège, pourquoi est-ce que tu ne t'en vas pas alors?", rétorque d'un air effronté le caloiro Tig. Jusque-là, le rituel se cantonne aux échanges langagiers où, non sans panache mais à ses dépens, "vain[cu] facilement le nouveau-venu" de l'avis même des autres "anciens" postés en spectateurs.

La rupture de ce jeu pourtant encadré conduit à un corps à corps entre les deux collégiens. Un corps à corps d'ailleurs ambigu, entre violence et attirance, à la faveur du fagging. En dépit de cet enchaînement - et c'est la seconde raison - la confrontation directe est médiatisée par le code strict qui régit la boxe. Ce "sport" de gentlemen relève du registre culturel en vigueur parmi l'élite scolarisée anglo-saxonne puisqu'il est, comme le précise Pessoa en note, l' "unique moyen de lutte parmi les garçons anglais. Celui qui se bagarre d'une autre façon est traité de lâche". Cette distinction sociale se vérifie à Barrowby: des trois garçons les plus avancés dans le cursus scolaire, l'un est la progéniture d'un lord 
et l'autre est un prince chinois, fils de mandarin. Ces statuts renforcent probablement leur "naturelle" emprise sur les autres. Toutefois ce critère social a sans doute un effet restreint sur les plus jeunes puisque les interrelations entre collégiens sont d'abord réglées par des codes endogènes à la communauté scolaire d'appartenance.

En ne s'arrêtant qu'au seul appendice nasal dans son approche physionomiste du texte pessoen, Jerónimo Pizarro nous paraît forcer son explication biographique, en référant le stéréotype alors courant qui associe un nez proéminent et l'origine juive de l'un des personnages, Zacharias au "nez de cigogne", et par extension ou identification du propre poète qui a pu revendiquer une telle généalogie ${ }^{19}$; il restreint du même coup le volet des items énoncés dans le texte. A contrario, parmi les trois expressions qui contiennent le mot "nez", deux d'entre elles s'accompagnent d'un complément "qualificatif" animalier (cigogne, rhinocéros), si fréquent parmi les élèves. Toutes ces connotations prennent place dans l'espace interactif d'un rite de praxe. La référence à deux physionomistes du XIXe siècle, Lavater et Kisch, survient uniquement avant cet interface ritualisé, au moment de la description par l'"auteur" Adolph Moscow des traits physiologistes de Mel, l'un des vétérans. Dans ce cas, les comparaisons zoomorphiques d'allure "descriptive" ne se font jamais par analogie entre lui et le règle animal. Une telle corrélation sert à définir exclusivement les newbies et ne survient que dans un cadre ludique. On a là une preuve d'un langage qui, parce que ritualisé, a pour effet de neutraliser le sens littéral des mots; il accompagne des actes euxmêmes formalisés. Lorsque, comme ici, il s'agit d'appliquer les principes de la physionomie à Mel, la sphère de référence des analogies est, non pas animalière, mais humaine, puisant aux registres psychologique et social: Mel possède ainsi "un caractère propre à un véritable garçon anglais; n'importe quel disciple habile de Lavater pouvait lire sur son visage les signes du courage et de l'espièglerie; dans les yeux bleus de la franchise et, s'il était disciple de Kisch, de la force de caractère et de la volonté et cette détermination particulière aux Anglais dans ses cheveux blonds".

Tout se passe comme si Jerónimo Pizarro soustrayait le nez du corps du texte, comme on isole arbitrairement le nez au milieu du

${ }^{19}$ Ce qui n'interdit pas que Pessoa, comme le soutien (mais en tant qu'idée exclusive) J. Pizarro, soit dès ces années intéressé, sinon préoccupé, par des questions relatives à la physiognomonie, notamment dans le rapport folie/génie; la preuve en est qu'il cite deux des précurseurs de cette "science": Lavater et Kisch (curieusement, c'est un homonyme, Marvell Kisch, qui est l'auteur de Os milhões de um doido, un autre "roman" inachevé). 
visage. Alors que cet organe ne fait véritablement signe que par rapport à l'ensemble narratif ou, pour poursuivre l'analogie, en fonction des traits du visage. On ne saurait donc prélever cet élément de la dramatisation rituelle présente dans le texte, où il fait sens et où il est efficient. En outre, cédant au réalisme que transcende pourtant le style romancé de cet écrit, Pizarro oublie le fait que la logique de la moquerie, si elle s'appuie parfois sur l'apparence physique de la victime, elle la déborde et la dépasse grâce à la dynamique de l'interaction et à l'inventivité langagières, dont on a plusieurs exemples imagés et vivants: "Eh toi, nez de proue de galère romaine!", "Morceau de tripe pourri!". L'esprit créatif des adolescents rejoint assez vite l'attitude caricaturante, verbale ou graphique. Cet art du portrait introduit à l'égard du réel un écart déformant, tant de nature quantitative (proportions exagérées du corps), que qualitative (défauts imputés aux mœurs et à l'intellect). Quand ne sont pas inventées, depuis le Moyen Age et dans tous les milieux étudiants d'Europe et d'ailleurs, des tares et des excroissances attribuées aux "nouveaux" et que les "anciens" s'activent à supprimer.

De la même façon, l'humour qui fuse dans des bons mots ou dans des jeux de mots grotesques, ne se limite pas au seul humour anglais dans lequel pointent des situations amusantes et un nonsense, comme le soutient António Sabler. Ce ton spirituel à la Dickens (doublé de paradoxes à la L. Carroll) se vérifie plus particulièrement dans le premier chapitre, où le prosateur dit son impossibilité de situer le collège du village de Barrowby, que pourtant il décrit l'un et l'autre dans le moindre détail; les cartographes ont de leur côté oublié de préciser les coordonnées d'un tel lieu. Et si seuls les topographes en ont dessiné le paysage alentour (dépourvu de centre), celui-ci n'a peut-être jamais existé, selon le narrateur. La présence d'un ton plaisant n'évacue pas d'autres pistes explicatives qui vont bien au-delà de la question du style. L'impossibilité de repérer dans le réel l'espace de l'action de Os apazes de Barrowby, ne provient-il pas d'un motif proprement littéraire, et singulièrement pessoen, qui dépasse le seul trait expressif? Cette raison, qui s'annonce dès 1903, est le refus du Réel donné, comme degré zéro de l'art, au profit d'une écriture portée par la puissance d'une imagination créatrice et d'une activité rêveuse, qu'illustreront avec maestria le Livro do Desassossego et Mensagem. La parole écrite invente "une réalité plus précieuse et splendide", comme dans le monde ludique des enfants. L"'horreur de la Réalité" s'oppose intentionnellement, pour un Pessoa qui est doté d'une "sensibilité si à fleur de peau", à une "artificialité 
plus divine", revendiquée par le poète fingidor dans bien des écrits, et notamment dans une belle "lettre à un poète" inconnu ${ }^{20}$.

En revenant au langage humoristique, nous pouvons remarquer qu'il est un autre type de plaisanterie prisé par Pessoa dès ses tendres années, et jamais repéré. Il s'inspire d'abord de la culture proprement scolaire. La rhétorique de nos jeunes collégiens, si elle est d'allure relâchée, n'en traduit pas moins le poids des apprentissages lettrés. Cette influence fait place à une appropriation créative de la part de la communauté adolescente, à travers pastiches et inversions parodiques, d'ordre comportemental ou verbal. On le voit lorsque, à une question du caloiro Zacharias, le vétéran Gyp rétorque: "Ah! Eh! Ih! Oh! Uh!". Cette "réponse" n'est pas si incongrue qu'elle en a l'air, puisqu'elle convoque les voyelles de l'alphabet, sorte de $b . a-b a$ par quoi les "anciens", jouant les pédagogues, initient aux "lettres" les néophytes réputés analphabètes. Pour l'heure, ces derniers sont ramenés rituellement par les aînés à un double seuil symbolique: celui anthropologique de l'humanité, en étant comparés (comme dans toutes les praxes depuis des siècles) à l'animalité et à l'enfance; et celui matériel et culturel des "humanités", en étant assimilés à des êtres incultes qui n'ont guère le droit de cité à l'école. Ces deux régressions symboliques, vers l'animalité sauvage et vers l'in-fans balbutiante et ignorante, se vérifient dans la multiplication de noms et de surnoms qui reflètent un riche bestiaire -et dont certaines espèces, comme l'âne et ses déclinaisons (âneries, bonnet d'âne, ânonner) ou des variantes telles que "bête" ou "oie", constituent un autre registre lié à la culture scolaire: “'Alors il y a de nouveaux garçons?', demanda Chine. 'Il y a ces trois-là', répondit Gyp; 'l'un à une face d'âne, l'autre un nez de cigogne et le troisième une bouche de requin pourri'"; “"Comment appelles-tu ceux-là?', demanda Gyp à ses camarades", en dévisageant les trois novatos. "Bêbêtes' (Bestinhas), répondit Mel avec un geste de dédain. 'Gamins' (Criancinhas) $)^{21}$, fut l'explication de Chine". Outre l'animalisation des nouveaux, ces termes collectifs ont pour effet de les désindividualiser, voire de les dépersonnaliser puisque, comme dans les pratiques ludo-rituelles étudiantes, les caloiros ne sont jamais interpellés par leur vrai nom. Sauf au terme du processus d'initiation, où un baptême symbolique marque leur incorporation à la société étudiante qui les a

${ }^{20}$ Citations tirées de cette lettre, in F. Pessoa, Páginas de estética e de teoria e crítica literárias (Lisbonne, Ática, s.d. - édition de G.R. Lind et J. do Prado Coelho) 134-135.

${ }^{21}$ Outre le rire, l'emploi de diminutifs est un autre exemple de la violence adoucie des rituels, ou d'une ritualisation de la violence. 
humanisés. Mais, le texte d'Adolph Moscow, inachevé, laisse en suspens la phase finale de la "réconciliation", qui n'est pas moins formalisée que l'étape des exacerbations et des soumissions.

Importance pour importance, la question des rites et des initiations sera par la suite autant, sinon plus souvent et plus longtemps, explorée par Pessoa que la "science" physiognomonique. Elle surgit très tôt, même si plus tard elle se déploiera sur un autre plan: celui de l'ésotérisme, de la kabbale, de la théosophie, de la franc-maçonnerie ou encore du messianisme sébastianiste. Du reste, entre l'initiation ésotérique et l'initiation scolaire, il existe une homologie structurale en termes de hiérarchieet de langage, comme aussi de finalité: l'enchaînement néophyte/ adepte/maître qui conduit aux vérités hermétiques a pour équivalent le lien novice/vétéran/maître. Dans ce dernier cas, les pôles novice/"ancien" sont unis, jusque dans leur opposition ritualisée et provisoire, dans une même entité par laquelle passe la transmission réservée (à une élite) et rationalisée du savoir scolaire, lequel est devenu pour ainsi dire une variante exotérique de la connaissance ésotérique. Pessoa ne dit pas autre chose lorsqu'il conçoit deux modalités de transmission des "Mystères": la voie exotérique ou profane qui concerne les enseignements "exposés de façon à ce que tous puissent être administrés" et la voie ésotérique ou occulte des apprentissages "plus véritables", réservée à "des individus préalablement préparés, préparés par degrés, près à les recevoir", ajoutant que "cette préparation s'appelle initiation" 22 .

\section{Message: une référence iconique et politique}

Le troisième et dernier fil qui relie Pessoa à Coimbra a pour support Mensagem. Le régime salazariste intègre cet ouvrage à sa propagande pédagogique en le faisant circuler dans les milieux scolaires et lettrés, tout en incrustant certaines de ses figures symboliques dans le décor artistique, d'allure totalitaire, de la Cité Universitaire.

${ }^{22}$ P. Teixeira da Mota, 'Esoterismo', in F. Cabral Martins (coord.), Dicionário de Fernando Pessoa e do Modernismo português, Lisbonne, Caminho (2008) 251. Dans un écrit intitulé Ensaio sobre a Iniciação, Pessoa établit une analogie entre la progression dans la connaissance des mystères, qui va du néophyte à l'adepte et au maître, et les degrés de la poésie, depuis l'apprentissage des "éléments culturels" (grammaire, culture générale, culture littéraire), jusqu'à la poésie dramatique qui fusionne tous les autres genres intermédiaires: $c f$. Y.K. Centeno, Fernando Pessoa e a filosofia hermética (Lisbonne, editorial Presença, 1985) 70. 


\section{L'appropriation de Message par le milieu lettré}

Seul ouvrage publié par le poète en portugais, sa langue patrie, Message est marqué par une épopée d'horizon téléologique. Il est couronné en décembre 1934 par le prix Antero de Quental dans la catégorie "poème". Cette récompense est attribuée par un organe ministériel du régime, le Secrétariat de la Propagande Nationale dirigé par un ancien d'Orpheu, António Ferro. Elle est remise au mois de février en la présence de Salazar... mais en l'absence du lauréat. José Augusto Seabra résume bien la position aujourd'hui partagée par nombre de pessoens: "Si l'intention patriotique de Message était évidente", jusque dans le titre initialement prévu, "Portugal", changé in extremis, et la date de diffusion, le ler décembre, ces caractéristiques jointent au contexte de l'Estado Novo ont conduit très tôt à des "équivoques et malentendus autour d'une œuvre qui ne relevait pas, comme certains l'ont aussitôt prétendu, du nationalisme dans le sens idéologique et politique du terme, mais d'une spiritualité universaliste, exprimée justement par le nouveau titre" qui renvoie, au moyen d'une pluralité d'anagrammes possibles, aux registres ésotérique et initiatique ${ }^{23}$.

La trame (trans)historique de nature emblématique et mystique de Message ainsi que sa reconnaissance officielle, font de ce long poème une œuvre rapidement connue, enseignée dans les écoles (une édition originale est présente au lycée de Coimbra), citée et réduite à quelques formules lyriques. On peut en donner deux exemples. Le premier concerne l'année 1937, quand le président de l'Association étudiante de Coimbra (AAC), le premier à être nommé contre la tradition par le régime, termine son allocution d'accueil d'étudiants brésiliens, par ces vers en vogue tirés du poème Mer portugaise "du poète" (sans le citer nommément): "Ô mer salée, combien dans ton sel tu contiens/De larmes versées par le Portugal!" 24 . Ce poème est d'abord publié en 1922 dans Contemporânea, puis en 1933 dans Revolução, la revue du fasciste Rolão Preto; il finit, en étant réajusté à la totalité organique, dans le recueil primé de 1934. Le second exemple s'enracine dans un contexte historique tout différent puisqu'il se rapporte à une récita de despedida actuelle qui se déroule

${ }^{23}$ J.A. Seabra, 'Para a história do texto pessoano: dos biografemas à heteronímia', in F. Pessoa, Mensagem, Poemas esotéricos (Madrid, Fundação eng. A. Almeida/ UNESCO, 1993) 211-212.

24 "O [sic] mar salgado, quanto ao [sic] teu sal/São lágrimas de Portugal!", Estudos, juin-juillet (1937) 386. 
en mai, durant la semaine festive de la Queima das fitas. Une récita est une sorte de manifestation culturelle d'adieu organisée par les finalistas de licenciatura, et instituée au milieu du XIXe siècle. Elle comporte une saynète écrite et jouée par les étudiants, accompagnée parfois d'une déclamation poétique et de canções de Coimbra. En mai 2008, les élèves de lettres ont interprété une Queima da Ode d'allure pessoenne, mais revue et corrigée à travers un esprit inventif: en recourant à la parodie, ils ont réalisé une "déclamation publique de l'Ode triomphale coïmbroise, suivie du brûlage de celle-ci, devant la Faculté de lettres à 13h01"; l'affiche qui annonçait le mini-spectacle se terminant par la formule: "Dieu veut, l'homme déclame, l'Ode brûle. Fernando Pessoa", une allusion humoristique au fameux incipit du poème "L'Infant" de Message: "Dieu veut, l'homme rêve, l'œuvre naît".

\section{La "Glorification du Génie Portugais"}

La Faculté de Lettres de Coimbra est achevée en 1951, dans le prolongement du bloc regroupant la Bibliothèque Générale et les Archives. Ces unités monumentales, aux lignes austères et orthogonales, constituent les premières constructions de la Cité Universitaire, un projet architectural conçut par Salazar dès 1937 et mis en place à partir de la décennie suivante. Cette esthétique totalitaire ${ }^{25}$, inspirée des plans architectoniques des fascismes européens (Allemagne, Italie, Espagne), est résolument antimoderniste car elle rejette les démarches des avantgardes cosmopolites "dénationalisées", incarnées par Pessoa et les "fous" d'Orpheu. Dans le même temps, cet art du pouvoir récupère, mais comme symbole dévoyé et appauvri, des références "nationalistes" de Message.

En 1951, deux fresques décoratives, réalisées dans le grand hall d'entrée de la nouvelle Faculté de Lettres de Coimbra, parachèvent sa construction. Celle de droite est baptisée par la Commission des travaux de la Cité Universitaire: "Allégorie de la Glorification du Génie Portugais"26. Mêlant l'allusion historique et la symbologie politique, elle est chargée de transmettre visuellement aux universitaires et aux visiteurs une image didactique de grandeur du Portugal, en faisant se

${ }^{25}$ N. Rosmaninho, O poder da arte. O Estado Novo e a Cidade Universitária de Coimbra (Coimbra, Imprensa da Universidade, 2006).

${ }^{26}$ Elle a été reproduite et décrite par M.D. Duarte, Faculdade de letras da Universidade de Coimbra: Ícone do Poder. Ensaio iconológico da imagística do Estado Novo (Câmara Municipal de Coimbra, 2003) 86-125. 
télescoper le passé national et le présent impérial. La fonction de cette iconographie, comme d'autres réalisations esthétiques localisées dans la Faculté de lettres (et ailleurs), déborde le seul criterium de l'art, pour s'inscrire dans une double perspective encomiastique: l'une, globale, d'exaltation du régime fasciste et ses valeurs nationalistes, qui se déploient dans une représentation du pouvoir et se prolongent dans un pouvoir de la représentation; l'autre, plus spécifique, de célébration de l'Université de Coimbra corporatiste et "humaniste"27. Cette perspective attribue une finalité instrumentale à l'art en étant investi idéologiquement par le champ politique. L'histoire nationale acquiert alors un contour et un contenu nationalistes, puisqu'elle sert une intention tactique et centralisée de l'Etat. La palette des "Génies Portugais" sélectionne surtout - mais pas exclusivement - des personnages "politiques": fondateur de la nation, rois, vice-roi des Indes, hommes d'Etat, bâtisseurs, navigateurs; ces figures avoisinent des objets emblématiques: glaive-croix, caravelles, sphère armillaire, cartes de navigation, écusson national. D'autres personnages renvoient par suggestion à cet horizon nationaliste: le Camões des Lusiades, l'Alexandre Herculano de l'Histoire de Portugal, le Gil Vicente en tant que père du théâtre portugais, le João Pinto Ribeiro patriote de la légitimation de la souveraineté nationale reconquise. D'autres aspects sont associés à la période fondatrice ou à celle des Découvertes et ajoutent une aura de sacralité à ces événements: Pedro Nunes mathématicien-cosmographe, Autel de la patrie, Sé Velha de Coimbra, pupitre de l'église Santa Cruz de la ville...

Dans ce cadre scénographique, une valorisation d'un genre différent semble se manifester. Elle n'a jamais été signalée. Cette stratégie recourt à une sorte d'allégorie mise en abyme, cette fois à une échelle locale: la Faculté de lettres, la première dans l'ordre hiérarchique des Facultés, paraît céder à une autocélébration. Cette stratégie est visible à plusieurs niveaux. D'abord à la localisation de la fresque des "Génies Portugais" au sein de cette Faculté, alors que son contenu imagistique transcende l'univers du savoir en général et les marques culturelles et identitaires relatives

${ }^{27}$ Chez les intellectuels de l'Estado Novo, de Salazar au catedrático et reitor Guilherme Braga da Cruz, ce terme a un sens précis: il réfère la formation intégrale de 1'Homme, c'est-à-dire celle d'une élite formée, sinon formatée, au plan indissociablement culturel, spirituel (catholique) et idéologique (nationalisme), avec le rejet des simples connaissances sans âme et de la technique du monde moderne qui favorisent le matérialisme. 
aux seules lettres. Nous obtenons une vérification supplémentaire si l'on remarque que toutes les autres réalisations artistiques de cette Faculté reflètent la spécificité "des lettres": la tapisserie de la salle du Conseil de la Faculté a été conçue pour représenter la science, l'art, la philosophie et la religion, au moins dans le projet initial; les deux motifs situés dans le hall de l'édifice matérialisent la "lecture" et l'"écriture"; les éléments décoratifs fixés sur la porte d'entrée principale font allusion, au moyen d'ornements stylisés, à de grands écrivains; enfin, les quatre statues placées sur l'esplanade d'accès incarnent l'éloquence, la philosophie, l'histoire et la poésie. Un autre argument - jamais non plus repéré - témoigne en faveur de cette autocélébration communautaire: ne peut-on voir, parmi les écrivains, historiens et artistes dessinés sur la fresque du hall d'entrée et qui portent un habit de ton bleu, la manifestation subtile de la couleur identitaire - le bleu foncé - de la Faculté de lettres (incluant notamment l'histoire et l'art), où certains se sont d'ailleurs formés?28

En scrutant de près la fresque "Glorification du Génie Portugais", Marco Daniel Duarte ${ }^{29}$ décèle entre cette peinture de teneur idéologique et Mensagem de Pessoa une similitude de ton et des références nominales communes (au nombre de sept). Ces noms personnifiés constituent un panthéon de héros nationaux sanctifiés. Ce panthéon est obtenu à l'aide d'allégories, pour le peintre, et de symboles, pour le poète. Coöncidence ou convergence intentionnelle, toujours est-il que plusieurs noms de rois et de héros portugais présents dans Message se retrouvent sur la fresque; de même qu'il existe une proximité entre l'idéalisation de l'histoire lusitanienne entreprise par Pessoa et le programme pictural des "Génies Portugais". La différence des styles et des intentions n'enlève rien, selon l'historien, au fait que "en dernière analyse, les objectifs se touchent"

${ }^{28}$ Parmi ces personnages, retenons: Almeida Garrett, Oliveira Martins, João Pinto Ribeiro, Machado de Castro, Luís de Camões. La plupart sont représentés dans une position basse (sur le plan du sol), marquant une opposition symbolique avec les valeurs politiques et spirituelles "élevées" (à tous les sens) incarnées dans des êtres et des objets-emblèmes, tels que Dom Afonso Henriques ou Saint Antoine, ou bien à une autre hauteur intermédiaire, le Padre António Vieira (prêchant sur un pupitre) ou les deux emblèmes de la nation que sont l'allégorie de la patrie et l'écusson national, placés au centre et sur un promontoire - le tout, comme le signale M.D. Duarte, est couronné par le glaive-Croix, brandit au sommet par le béatifié Nuno Álvares Pereira.

${ }^{29}$ M.D. Duarte, 'Os Símbolos da 'Mensagem' de Fernando Pessoa nos Frescos da Faculdade de Letras da Universidade de Coimbra', Comunidade de Lingua Portuguesa, São Paulo, Centro de Estudos Fernando Pessoa (2007) 38-52. 
car ils s'inspirent d'un même "substrat culturel fait de mythes, de héros, de symboles, de signes et de signaux" ${ }^{30}$. L'auteur parle néanmoins à diverses reprises des divergences entre les deux artistes et la qualité de leurs œuvres respectives, l'une étant transhistorique, tandis que l'autre est circonscrite à son époque; de même que les contextes de ces deux créations ne sont guère superposables. Par-delà les moyens, les buts et les phases historiques, il faut pourtant davantage insister sur le point essentiel qui les sépare, pour éviter le risque de céder à une comparaison empirique superficielle: le peintre, qui soumet à une volonté supérieure les thèmes et les objectifs d'un projet qui n'est pas le sien, reste reclus dans le cadre pauvre et étroit d'une échelle nationale et d'un nationalisme passéiste qui se dote de "traditions inventées" (E. Hobsbawm), là où Pessoa inscrit sa démarche, qui commande jusqu'au contenu de son poème - dont la pluralité de sens continue de faire l'objet de multiples interprétations -, dans un nationalisme cosmopolite de poète en devenir, spirituel et mystique, qui ouvre à un horizon universel.

Si le nom de Pessoa n'est pas incorporé à cette galerie de portraits tournée vers un passé idéalisé et esthétisé, il apparaît tout près de là, sur la porte d'entrée ouvragée de la Faculté de lettres. En effet, à côté d'Homère, de Gil Vicente et de Camões, un portique est réservé à six poètes contemporains. Fernando Pessoa est l'un d'eux, étant comme les autres repérable de façon métonymique, à travers un motif en métal qui représente un élément jugé central de son œuvre: un mostrengo. De toute l'œuvre pessoenne, n'est retenu à nouveau que Message; cette réduction se double d'une autre puisque, parmi les riches références que renferme cet ouvrage, seule une image en est extraite, peut-être à cause de son équivalent chez Camões. Entre art, histoire et idéologie, l'identité convoquée - et instrumentalisée - de Pessoa joue alors sur un double tableau d'une réception sociale où se croisent de façon stratégique le politique et l'esthétique: une héroïsation par le pouvoir du poète, qui est de ce fait consacré par l'histoire nationale dans sa version nationaliste; et cette fois au bénéfice de la dictature, une légitimation littéraire d'un passé glorifié grâce au ton patriotique et messianique de Message ${ }^{31}$.

${ }^{30}$ M.D. Duarte (art. cité) 42 et 52.

${ }^{31} \mathrm{~L}$. Reis Torgal signale la diffusion par le régime salazariste, à travers collections et bibliothèques "populaires", de toute une "galerie de héros, de martyrs et de saints" qui recoupe celle présente dans la fresque des lettres; il ajoute, à propos de l'ouvrage Message de Pessoa, qu'il alimente "le sentiment épique des lecteurs, servant de ciment à la croyance sébastianiste d'une salvation nationale que l'Estado Novo a pensé 


\section{Conclusion}

Notre étude sur Fernando Pessoa a tenté d'éclairer deux questions inexplorées qui ont pour cadre le milieu lettré de Coimbra. Dans un mouvement circulaire entre les écrits de jeunesse du poète et le contexte culturel et politique de Coimbra, nous avons appréhendé son œuvre proto-hétéronymique à partir de deux angles distincts, quoique entrecroisés: une présence littéraire de Coimbra et de ses traditions étudiantes dans la prose de Pessoa; et une projection artistique, opérée à des fins de propagande, de Message, qui est stratégiquement lu par le régime salazariste comme un texte nationaliste.

Sous l'Estado Novo, l'espace universitaire de Coimbra est devenu le théâtre d'une représentation idéologique du Portugal. Cette opération esthético-politique a recouru à un détournement du sens de Message, qui a fait l'objet entretemps, et au-delà même de l'Estado Novo, d'une "nationalisation" et d'une patrimonialisation. Le refus du poète d'Orpheu d'enseigner à Coimbra ne l'a pas empêché de hanter la Alta, de façon posthume et transfigurée. Mais cette gloire-là sans universalité, il l'a récusée très tôt. Dans une lettre à sa mère (5/6/1914), il s'interroge: "Que serai-je moi d'ici à dix ans?[...] Peut-être que la gloire sent la mort et l'inutilité, et que le triomphe a un goût de pourriture".

\section{Bibliographie}

Centeno, Y.K., Fernando Pessoa e a filosofia hermética, Lisbonne, Editorial Presença, 1985.

Costa, E. Freitas da, Fernando Pessoa. Notas a uma biografia romanceada, Lisbonne, Guimarães, 1951.

Duarte, M.D., 'Os Símbolos da 'Mensagem' de Fernando Pessoa nos Frescos da Faculdade de Letras da Universidade de Coimbra', Comunidade de Lingua Portuguesa, São Paulo, Centro de Estudos Fernando Pessoa (2007) 38-52.

interpréter. De là les fréquentes citations de Message au cours de discours officiels et l'attribution à cette œuvre, en 1934, du prix Antero de Quental, institué par le SPN": cf. L. Reis Torgal, História e ideologia (Coimbra, Minerva-História, Livraria Minerva, 1989) 187 et 191-192. 
Duarte, M.D., Faculdade de letras da Universidade de Coimbra: Ícone do Poder. Ensaio iconológico da imagística do Estado Novo, Câmara Municipal de Coimbra, 2003.

Frias, A., Le monde universitaire et la Praxe académica au Portugal. Traditions étudiantes et cultures académiques à l'Université de Coimbra (thèse de doctorat d'ethnologie), Université Paris X Nanterre, 2 vols, 2003.

Jennings, H.D., Os dois exílios. Fernando Pessoa na Africa do Sul, Porto, Centro de Estudos Pessoanos, s.d. [1984].

Lopes, T. Rita, Pessoa por conhecer, vols. I et II, Lisbonne, Editorial Estampa, 1990.

Martins (coord.), F. Cabral, Dicionário de Fernando Pessoa e do Modernismo português, Lisbonne, Caminho (2008): entrées 'Diabo Azul', 'Esoterismo', 'Moscow, Adolph'.

Nogueira M., 'A influência de Henrique Rosa em Fernando Pessoa e o mistério do Diabo Azul': in J. Pizarro (org.), Fernando Pessoa: o guardador de papéis, Lisbonne, 2009.

Pessoa, F., Escritos autobiográficos, automáticos e de reflexão pessoal (édition de R. Zenith), Lisbonne, Assírio e Alvim, 2003.

Pessoa, F., 'Os Rapazes de Barrowby', Persona 9 (1983) 50-65.

Pessoa, F., Páginas de estética e de teoria e crítica literárias (édition de G.R. Lind et J. do Prado Coelho), Lisbonne, Ática, s.d. [1966].

Pizarro, J., Fernando Pessoa: entre génio e loucura, Lisbonne, IN-CM, collection "Estudos", vol. III, 2007.

Rodrigues (éd.), M.A., A Universidade de Coimbra no Século XX, Coimbra, Publicações do Arquivo da Universidade de Coimbra, 1988.

Rosa, J. Mendes, Pad'Zé: o cavaleiro da utopia, edição da Junta de Freguesia de Aldeia de Joanes, 2000.

Rosmaninho, N., O poder da arte. O Estado Novo e a Cidade Universitária de Coimbra, Coimbra, Imprensa da Universidade, 2006.

Sabler, A., 'The man who liked Dickens', Persona 9 (1983) 47-49.

Seabra, J.A., Fernando Pessoa ou o Poetodrama, Lisbonne, IN-CM, 1988 (1ère éd. 1974).

Seabra, J.A., 'Para a história do texto pessoano: dos biografemas à heteronímia', in F. Pessoa, Mensagem, Poemas esotéricos, Madrid, Fundação eng. A. Almeida/UNESCO (1993) 191-215.

Simões, J. Gaspar, Vida e obra de Fernando Pessoa, Lisbonne, Livraria Bertrand, 1950 (4e éd. 1979).

Faculdade de Letras | Universidade de Coimbra 
Torgal, L. Reis, História e ideologia, Coimbra, Minerva-História, Livraria Minerva, 1989.

Vieira, Y. Frateschi, Sob o ramo da bétula. Fernando Pessoa e o erotismo vitoriano, São Paulo, Editora da Unicamp, 1989. 